e.g., $1 \mu \mathrm{s}$, is used. One may be able to sweep the frequency of the laser over several $\mathrm{GHz}$ during a single pulse, using the demonstrated methods for frequency tuning with electrooptic or acoustooptic devices [8]-[10]. The possibility of making velocity measurements with temporal resolution less than $1 \mu \mathrm{s}$ at a repetition rate of $10^{4} / \mathrm{s}$ should be very interesting for certain applications such as turbulence measurements.

In conclusion, we have shown theoretically that stimulated Raman gain spectroscopy of moving molecular gases is a promising method for measuring flow velocities accurately and nonintrusively at sonic speeds, where current LDV methods begin to fail. The velocity resolution achievable should be on the order of Mach 0.04, with no limit on high speeds. We have proposed several methods by which a velocity measurement could be made in less than $100 \mathrm{~ns}$. with a single laser pulse. The measurement repetition rate is currently limited to about $10^{4} / \mathrm{s}$ by available laser repetition rates. It is also possible that species concentration [11], [12] and local temperature [13] measurements could be made at the same time. We note that CARS and other coherent Raman spectroscopy techniques [2] could also be used and, in many cases, should give competitive signal-to-noise.

\section{ACKNOWLEDGMENT}

The authors wish to thank A. Owyoung for providing a copy of a nitrogen SRG spectrum before its publication.

\section{REFERENCES}

[1] F. Durst, A. Melling, and J. Whitelaw, Principles and Practice of Laser-Doppler Anemometry. New York: Academic, 1976.

[2] M. D. Levenson, "Coherent Raman spectroscopy," Phys. Today, vol. 30, pp. 44-49, May 1977.

[3] A. Owyoung, "CW stimulated Raman spectroscopy," in Chemical Applications of Nonlinear Spectroscopy, Harvey, Ed. New York: Academic, to be published.

[4] --, "Coherent Raman gain spectroscopy using CW laser sources," IEEE J. Quantum Electron., vol. QE-14, pp. 192-203, 1978.

[5] - "High-resolution CW stimulated Raman spectroscopy in molecular hydrogen," Opt. Lett., vol. 2, pp. 91-93, 1978.

[6] C. W. Wieman and T. W. Hänsch, "Doppler-free laser polarization spectroscopy," Phys. Rev. Lett., vol. 36, pp. 1170-1173, 1976.

[7] G. L. Eesley, M. D. Levenson, and W. M. Tolles, "Optically heterodyned coherent Raman spectroscopy," IEEE J. Quantum Electron., vol. QE-14, pp. 45-49, 1978.

[8] I. M. Telle and C. L. Tange, "Very rapid tuning of CW dye laser," Appl. Phys. Lett., vol. 26, pp. 572-574, 1975.

[9] J. S. Gerig and H. Montague, "A simple optical filter for chirpped radar," Proc. IEEE, vol. 52, p. 1753, 1964.

[10] U. Hochuli, P. Haldemann, and S. Siahatgar, "Display of the 8521-A cesium hyperfine lines using a swept GaAs laser," J. Appl. Phys., vol. 40, pp. 3374-3375, 1969.

[11] P. R. Régnies and J. P. E. Taran, "On the possibility of measuring gas concentration by stimulated anti-Stokes scattering," Appl. Phys. Lett., vol. 23, pp. 240-242, 1973.

[12] A. C. Eckbreth, "BOXCARS: Cross-beam phase-matched CARS generation in gases," Appl. Phys. Lett., vol. 32, pp. 421-423, 1978.

[13] F. Moya, S. A. J. Druet, and J. P. E. Taran, "Gas spectroscopy and temperature measurement by coherent Raman anti-Stokes scattering," Opt. Commun., vol. 13, pp. 169-174, 1975.

\title{
The Intrinsic Electrical Equivalent Circuit of a Laser Diode
}

\author{
J. KATZ, S. MARGALIT, C. HARDER, D. WILT, AND A. YARIV
}

\begin{abstract}
The basic electrical equivalent circuit of a laser diode is derived. The effects of spontaneous emission and self-pulsations are included. It is found that self-pulsations are represented by a negative resistance in the model. Application of this model suggests purely electronic methods of suppressing relaxation oscillations in laser diodes.
\end{abstract}

$\mathrm{T}$ HE many applications of laser diodes, and in particular those which involve high frequency modulation or combined operation with electronic components and circuits, require an accurate representation of the electrical impedance

Manuscript received September 9, 1980. This work was supported by the National Science Foundation and the Office of Naval Research under the Optical Communications Program.

The authors are with the California Institute of Technology, Pasadena, CA 91125. characteristics of the laser. The elements of the laser equivalent circuit are derived from the rate equations [1], which describe the interplay between the optical intensity (or total number of photons) and the injected carriers. These equations are augmented by the voltage-carrier density characteristics of the injection junction.

Recently, using this approach, Morishita et al. [2] have shown that the laser diode can be modeled by a parallel $R L C$ circuit [see Fig. 1(a)] whose elements are

$$
\begin{aligned}
R & =R_{d}\left(I_{t h} / I^{0}\right) \\
L & =R_{d} \tau_{p h} /\left[\left(I^{0} / I_{t h}\right)-1\right] \\
C & =\tau_{s} / R_{d}
\end{aligned}
$$

where 


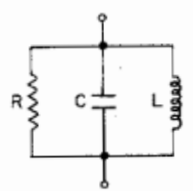

(a)

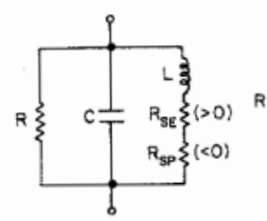

(c)

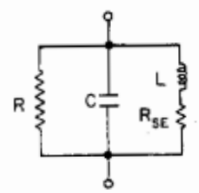

(b)

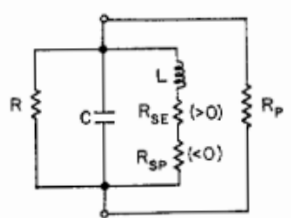

(d)
Fig. 1. Equivalent circuit of a laser diode: (a) basic model, no spontaneous emission; (b). model including the effect of spontaneous emission; (c) model including effects of spontaneous emission and self-pulsations; (d) laser diode with an externally connected resistor. Values of the components are given in the text.

$$
R_{d}=\frac{2 k T}{q} \frac{1}{I_{d}}
$$

is the usual expression of a differential resistance of a diode, $\tau_{s}$ is the spontaneous carrier lifetime, $\tau_{p h}$ is the photon lifetime, $I^{0}$ is the bias current of the laser diode, and $I_{d}$ is a

$$
\begin{aligned}
& \frac{d N_{e}}{d t}=\frac{I}{q a d}-A\left(N_{e}-N_{o m}\right) N_{p h}-\frac{N_{e}}{\tau_{s}} \\
& \frac{d N_{p h}}{d t}=A\left(N_{e}-N_{o m}\right) N_{p h}-\frac{N_{p h}}{\tau_{p h}}+\beta \frac{N_{e}}{\tau_{s}}
\end{aligned}
$$

where $N_{p h}$ is the photon density, $A$ is a proportionality constant, $N_{\text {om }}$ is the minimum electron density required to obtain a positive gain, and $\beta$ is the fraction of the spontaneous emission that is coupled the lasing mode $(\beta$ is a small number, of the order of $10^{-4}$ ). The assumptions that go into formulating (4) and (5) are that the laser oscillates in a single mode, the inversion is homogeneous, and the gain is linear in the difference between $N_{e}$ and $N_{o m}$ (and zero for $N_{e}<N_{o m}$ ). The junction voltage $V$ is introduced into the equation by relating the carrier density $N_{e}$ to $V$ by

$$
N_{e}=N_{i} \exp \frac{q V}{2 \mathrm{kT}}
$$

where $N_{i}$ is the intrinsic carrier density and $V$ is the junction voltage; the $q V / 2 \mathrm{kT}$ dependence is a good approximation for GaAs devices. A small-signal solution of (4)-(6) about the quiescent point leads to the following impedance function of the laser

$$
Z(\omega)=\frac{V(\omega)}{I(\omega)}=R_{d} \frac{i \frac{\omega}{\tau_{s}}+\frac{1+n_{o m}-n_{e}^{0}}{\tau_{s} \tau_{p h}}}{-\omega^{2}+i \frac{\omega}{\tau_{s}}\left[n_{p h}^{0}+1+\frac{\tau_{s}}{\tau_{p h}}\left(1+n_{o m}-n_{e}^{0}\right)\right]+\frac{n_{p h}^{0}+\beta\left[n_{e}^{0}\left(1+1 / n_{p h}^{0}\right)-n_{o m}\right]}{\tau_{s} \tau_{p h}}}
$$

normalized current given by

$$
I_{d}=\frac{N_{e}^{0}}{\tau_{s}} a q d
$$

where $N_{e}^{0}$ is the dc value of the injected electron density (in a p-type material), $a$ is the diode area, $d$ is the thickness of the active region, and $q$ is the electron charge. $I_{d}$ is approximately equal to the current in the diode below the threshold, and to the threshold current for currents above the threshold. In AlGaAs lasers, typical values of $R$ are about or less than 1 $\Omega$, values of $L$ are about $\mathrm{pH}$, and values of $C$ are about $3 \mathrm{nF}$. The model of Morishita et al. [2] does not take into account the physical phenomena of spontaneous emission into the laser mode and saturable losses (or equivalently, superlinear gains), and consequently fails to predict the occurrence of self-pulsations and its disturbing effect on the modulation frequency response. The purpose of this paper is twofold: first, to include the effect of spontaneous emission and saturable absorption (or gain saturation) in the equivalent circuit; second, to use the improved equivalent circuit to consider the possibility of modifying the frequency response of the laser diode by electronic means.

We first consider the effect of spontaneous emission. We do this by adding the term $\beta\left(N_{e} / \tau_{s}\right)$ to the photon rate equation (5). The first equation (4) remains unchanged. where $V(\omega)$ and $I(\omega)$ are the complex amplitudes of the small-signal junction voltage and current, respectively, and $n_{p h}^{0}, n_{e}^{0}$, and $n_{o m}$ are the normalized steady-state values of $N_{p h}^{0}, N_{e}^{0}$, and $N_{o m}$, respectively $\left(n_{p h}^{0}=A \tau_{s} N_{p h}^{0}, n_{e}^{0}=A \tau_{p h} N_{e}^{0}\right.$, and $\left.n_{o m}=A \tau_{p h} N_{o m}\right)$. In the case of $\beta=0, n_{p h}^{0}=\left(I^{0} / I_{t h}\right)-$ 1 if $I^{0}>I_{t h}$, and $n_{p h}^{0}=0$ if $I^{0}<I_{t h}$. In the general case when $\beta \neq 0, n_{p h}^{0}$ can be solved as a function of $I^{0}$ [1]. Typical values are about 0.1 at threshold and about 10 at very high current levels.

It is interesting to see that the impedance function of (7) is that of a parallel $R L C$ circuit with a resistor $R_{s e}$ in series with the inductance. The values of the components are [see Fig. 1(b)]

$$
\begin{aligned}
R & =R_{d} /\left(n_{p h}^{0}+1\right) \\
L & =R_{d} \tau_{p h} /\left(n_{p h}^{0}+\beta\right)\left(n_{e}^{0}-n_{o m}\right) \approx R_{d} \tau_{p h} / n_{p h}^{0} \\
C & =\tau_{s} / R_{d} \\
R_{s e} & =\beta R_{d} \frac{n_{e}^{0}}{n_{p h}^{0}\left(n_{p h}^{0}+\beta\right)\left(n_{e}^{0}-n_{o m}\right)} \approx \beta R_{d} \frac{n_{e}^{0}}{\left(n_{p h}^{0}\right)^{2}} .
\end{aligned}
$$

It should be noted that the approximated forms of ( $8 \mathrm{~b})$ and (9) become exact when $n_{\text {om }}$ equals zero. 


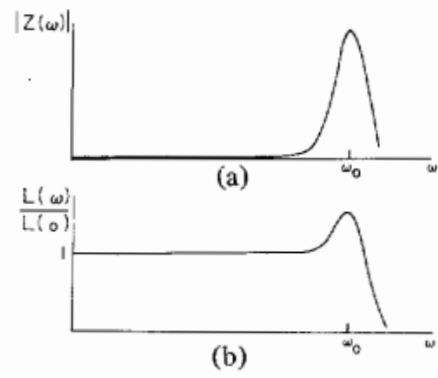

Fig. 2. (a) Impedance of a laser diode and (b) light versus current transfer function of a laser diode.

In order to understand the methods for modifying the light versus current transfer function of the laser diode, one has to compare that function to the impedance function given by (7). In Fig. 2 we compare the magnitude of the impedance $|Z(\omega)|$ with the magnitude of the normalized light response function $\left(|L(\omega) / L(0)|=\left|\left[n_{p h}^{1}(\omega) / I(\omega)\right] /\left[n_{p h}^{1}(0) / I(0)\right]\right|\right.$, where $L$ denotes the light output from the laser diode. Near the resonance frequency $\left(\omega_{0} \simeq\left[n_{p h}^{0} /\left(\tau_{s} \tau_{p h}\right)\right]^{1 / 2}\right)$, the two functions have the same shape; at lower frequencies the impedance is virtually zero (i.e., perfect gain clamping), but the light response is flat and equal to its dc value. Thus, any component connected in parallel with the laser diode will be effectively shorted, and its influence will be manifested only near resonance, where it is needed. For example, the effect of a resistor $R_{p}$ connected in parallel with the diode, as shown in Fig. 1(d), is to decrease the resonance peak by the ratio of $R$ to $R R_{p} /\left(R+R_{p}\right)$. Of course, resistors that are of practical values $(\widetilde{<} 1 \Omega)$ cannot be added in a dc fashion. One can either use ac coupling or other devices that have the desired value of differential impedance around the bias point, but without effectively shorting the dc current. Since the impedance is essentially zero below the resonance frequency, the relaxation oscillations can be controlled, in principle, by connecting a resistor across the diode instead of a tuned circuit [7].

The analysis of the effects of external electronic components (parasitic or not) connected to the laser diode is very straightforward. Since solving the rate equations for the ac impedance results in linearization of the equations, all external electrical components can be added directly to the equivalent circuit of Fig. 1(b), and thus their effect can be readily evaluated.

Self-pulsations can be accommodated in the model by including a saturable absorption term in the rate equation for the photons [3]

$$
\begin{aligned}
\frac{d N_{p h}}{d t}= & A\left(N_{e}-N_{o m}\right) N_{p h}+\beta \frac{N_{e}}{\tau_{s}}-\frac{N_{p h}}{\tau_{p h}} \\
& \cdot\left\{1+\frac{\delta}{\left[1+\left(N_{p h} / N_{s}\right)\right]}\right\}
\end{aligned}
$$

where $\delta$ is a parameter denoting the strength of the nonlinearity $(\delta \approx 0.02[3])$ and $N_{s}$ is the saturation density of the photons $\left(N_{s} \approx 10^{13}-10^{14} \mathrm{~cm}^{-3}\right.$ [3], [5] ). Performing small-signal analysis on (4), (6), and (10), we find that the effect of saturable absorption is to introduce in the equivalent circuit a negative resistance $R_{s p}$ which is in series with the inductance
[Fig. 1(c)]. The value of this resistance is

$$
\begin{aligned}
R_{s p} & =-\frac{R_{d} \delta}{n_{s}} \frac{1}{\left[1+\left(n_{p h}^{0} / n_{s}\right)\right]^{2}} \frac{n_{p h}^{0}}{\left(n_{p h}^{0}+\beta\right)\left(n_{e}^{0}-n_{o m}\right)} \\
& \approx-\frac{R_{d} \delta}{n_{s}} \frac{1}{\left[1+\left(n_{p h}^{0} / n_{s}\right)\right]^{2}}
\end{aligned}
$$

where $n_{s}$ is the normalized photon saturation density $\left(n_{s}=\right.$ $A \tau_{s} N_{s}$ ), and it is connected in series with the resistor describing the spontaneous emission. It has been found recently [4] that self-pulsations are accompanied by negative resistance of the laser. Also in an earlier work [6], it was found that oscillations in the light output from the laser occur simultaneously with oscillations in the current. It is interesting to note that phenomena that are optical (self-pulsations, spontaneous emission) are responsible for elements in the "optical" branch in the equivalent circuit (i.e., in series with inductance). Also, the effect of the spontaneous emission, modeled as a positive resistance $R_{s e}$, is to reduce the causes of self-pulsations, which are modeled by a negative resistance.

Fig. 3 shows some calculated results from the rate equations of the dc resistance of the laser diode near threshold for several values of $\beta$ and $N_{s}$. These graphs indicate large values of negative resistance near the threshold for small values of $\beta$ and $N_{s}$, and are in a good agreement with the experimental results reported in [4]. In this case there is also a superlinear behavior in the light versus current curve of the laser in a narrow region near the threshold. For larger values of $\beta$ and $N_{s}$, the absolute value of the dc resistance near threshold is small, and its sign depends on the particular values of the parameters. Generally, lasers with large $\beta\left(\sim 10^{-3}\right)$ do not possess negative resistance and thus are less likely to exhibit self-pulsations, and lasers with large values of $N_{s}$ tend to have negative dc resistance with a small absolute value, and thus are less influenced by external electronic circuits. In general, since the values of the components in the model depend on the operating point, the resistance can be negative in one region of bias currents and positive in the other. It should also be noted that if the dc resistance of the laser diode is negative, the real part of its impedance will be negative for all frequencies. Values taken for the calculations are $\delta=0.02, A=1.510^{-6} \mathrm{~cm}^{3} \cdot \mathrm{s}^{-1}, N_{\text {om }}=$ $5.10^{17} \mathrm{~cm}^{-3}, d=0.3 \mu \mathrm{m}, a=300 \times 5 \mu \mathrm{m}^{2}, \tau_{p h}=1 \mathrm{ps}$, and $\tau_{s}=3 \mathrm{~ns}$. The agreement between the results obtained by a direct solution of the rate equations and from the approximate electrical model is very good.

As a final remark we should note that parasitic electronic components, which are not intrinsic to the laser operation but are nevertheless always present, tend to reduce the effect of externally connected electrical networks. Among these components are the parasitic capacitance of the diode, and mainly the parasitic series resistance. In order to exercise a significant control by connecting external components in parallel with the diode, the condition $2 k T / q I_{\text {th }} \gg R_{c}\left(R_{c}\right.$ is the contact resistance of the diode) should be fulfilled. This condition is difficult to realize presently, but there is no reason why in future low threshold improved lasers this condition cannot be met.

In conclusion, we have presented an electrical equivalent 


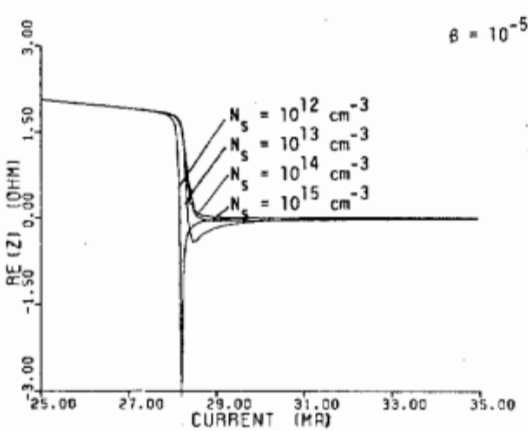

(a)

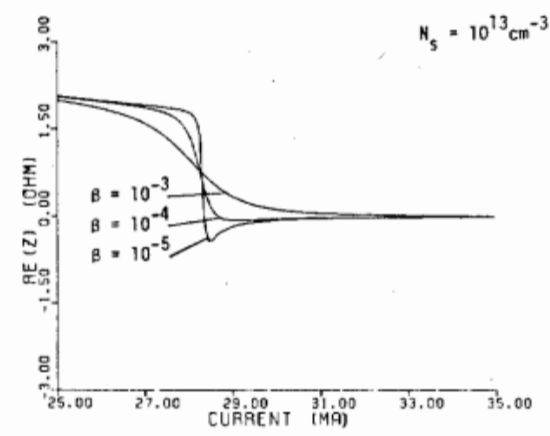

(c)

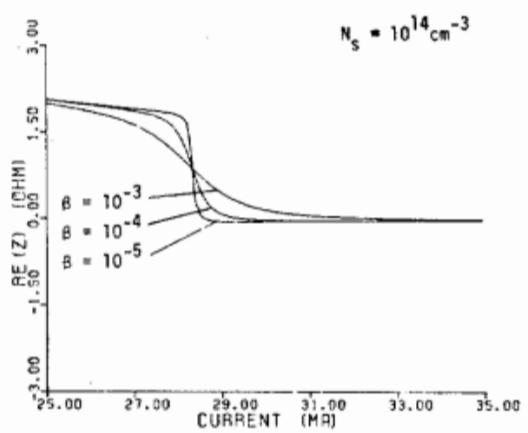

(b)

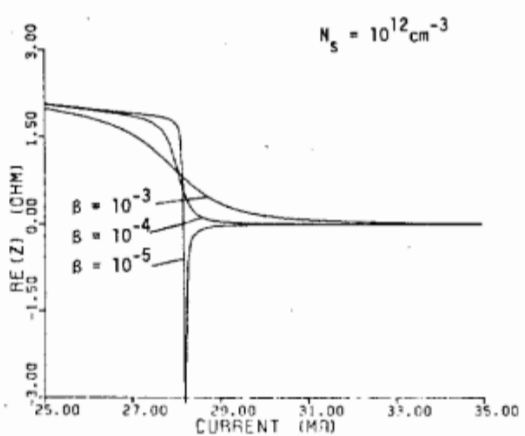

(d)

Fig. 3. The dc resistance of a laser diode. (a) $\beta=10^{-5}$; (b) $N_{s}=10^{14}$ $\mathrm{cm}^{-3}$; (c) $N_{s}=10^{13} \mathrm{~cm}^{-3}$; (d) $N_{s}=10^{12} \mathrm{~cm}^{-3}$.

circuit which describes the impedance of the laser diode, including the effects of self-pulsations, spontaneous emission, and external electronic components. Laser diodes with extremely low contact resistance are a prerequisite for effectively reducing the resonance in the light frequency response by external electronic means.

\section{REFERENCES}

[1] H. Kressel and J. K. Butler, Semiconductor Lasers and Heterojunction Leds. New York: Academic, 1977, ch. 17.

[2] M. Morishita, T. Ohmi, and J. Nishizawa, "Impedance characteristics of double-heterostructure laser diodes," Solid-State Electron., vol. 22, pp. 951-962, 1979.

[3] K. Y. Lau, L. Figuroa, and A. Yariv, "Generation and quenching of intensity self-pulsations in semiconductor lasers coupled to external cavities," presented at the 11th Int. Quantum Electron. Conf., Paper W.4, Boston, MA, June 23-26, 1980.

[4] P. J. Anthony, T. L. Paoli, and R. L. Hartman, "Observations of negative resistance associated with superlinear emission characteristics of (Al, Ga) As double-heterostructure lasers," IEEE J. Quantum Electron., vol. QE-16, pp. 735-739, July 1980.

[5] R. L. Hartman, R. A. Logan, L. A. Koszi, and W. T. Tsang, "Pulsations and absorbing defects in (Al, Ga) As injection lasers," $J$. Appl. Phys., vol. 50, pp. 4616-4637, 1979.

[6] L. A. D'Asaro, J. M. Cherlow, and T. L. Paoli, "Continuous microwave oscillations in GaAs junction lasers," IEEE J. Quantum Electron., vol. QE-4, pp. 164-167, 1968.

[7] Y. Suematsu and T. H. Hong, "Suppression of relaxation oscillation in light output of injection lasers by electrical resonance circuit," IEEE J. Quantum Electron., vol. QE-13, pp. 756-762, 1977. 